\title{
DESARROLLO DE PROYECTO PROGRESIVO DE VIVIENDA DE INTERES SOCIAL CON SISTEMAS TRADICIONALES E INDUSTRIALIZADOS
}

\author{
(DEVELOPMENT OF A PROGRESSIVE PROJECT ON HOUSING WITH SOCIAL INTEREST \\ USING TRADITIONAL AND INDUSTRIALIZED SYSTEMS)
}

EQUIPO DE PROYECTOS Y CONSTRUCCIONES G.E.G. LTDA.:

\begin{abstract}
Jorge Gafaro Briceño, Arquitecto
\end{abstract}
Gerente

Alberto Estrada Pulido, Arquitecto

Director Dpto. de Diseño

Juan B. Gómez Rodríguez, Arquictecto

Director Dpto. de Programación y Control

COLOMBIA

\section{RESUMEN}

Al afrontar el desarrollo de un proyecto arquitectónico para vivienda masiva, Proyectos y Construcciones G.E.C. considera que en el estado actual de desarrollo de la industria de la construcción en Colombia y su situación económica, lo más conveniente es continuar usando materiales y sistemas tradicionales, aplicándoles los de estudios, planeación y organización que son propios de cualquier industria de alta productividad, pero que entre nosotros se consideran ajenos a la construcción tradicional.

\section{SUMMARY}

On taking up the development of an architectural project for mass housing, "Proyectos y Construcciones G.E.G. considers that, in the present state of development of the building industry in Colombia and its financial situation, the most convenient thing to do would be to continue using traditional materials and systems, towhich would be applied those pertaining to research, planning and organization which are characteristic of any industry enjoying high output, but which are considered by us to be foreign to traditional construction.

\section{INTRODUCCION}

Labor realizada en los dos programas presentados a su consideración por nuestra firma: Aurora II y Marichuela.

a) Diseño Urbanístico.

b) Diseño Vivienda.

c) Dirección Arquitectónica.

Miles de nuevas Unidades de Vivienda

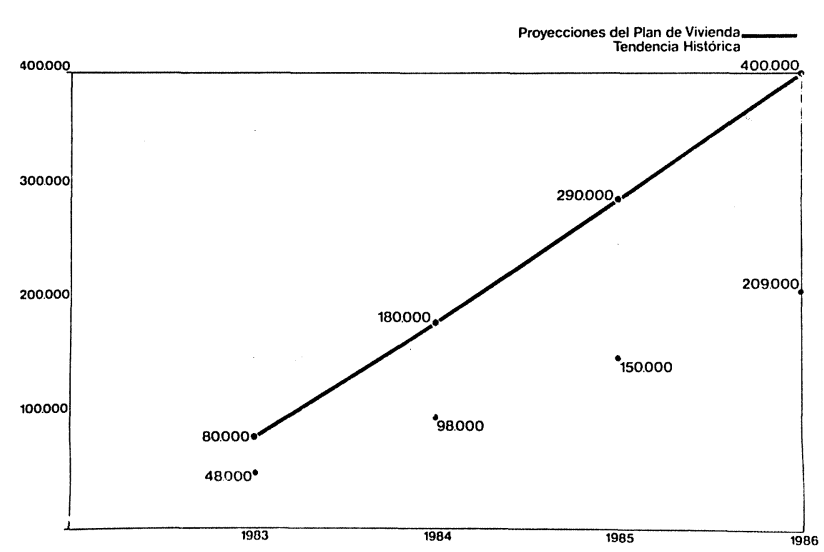

d) Programación general para la urbanización y para la vivienda.

e) Control semanal de la programación.

f) Asesoría en construcción.

Finalmente la construcción tanto de la urbanización y de las viviendas fue realizada por "Ingeniobras Bogotá Ltda.", firma independiente de la nuestra.

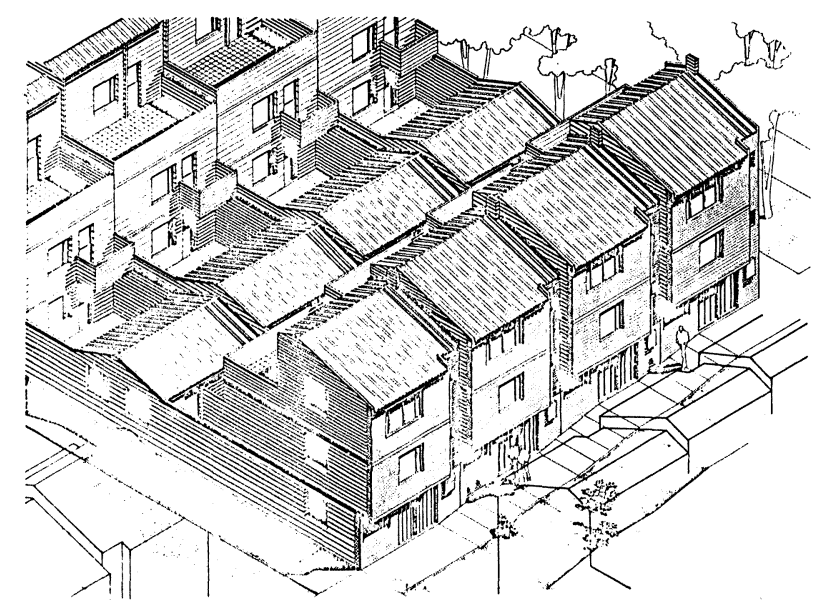

Proyecciones plan de vivienda y perspectiva. 


\section{LA VIVIENDA DE INTERES SOCIAL EN EL MARCO URBANO DE NUESTRA CIUDAD}

Una de las grandes consecuencias del subdesarrollo en los paises latinoamericanos, ha sido durante este siglo, el aceleramiento continuo del proceso de urbanización. En la Argentina y el Brasil este proceso estuvo controlado por la previsión, a tiempo, de estructuras urbanas sólidas que buscaban recibir los grandes fenómenos migratorios del Sur Europeo. Sin embargo, en el resto del continente los núcleos urbanos de primera importancia se han deformado en su planeación urbanística y en sus caracteristicas socioeconómicas, desbordando las posibilidades físicas de su situación geográfica.

En Colombia como caracteristica particular dentro del continente, el problema de la migración se produce sobre varios centros urbanos, hecho que no mengua la dimensión del problema.

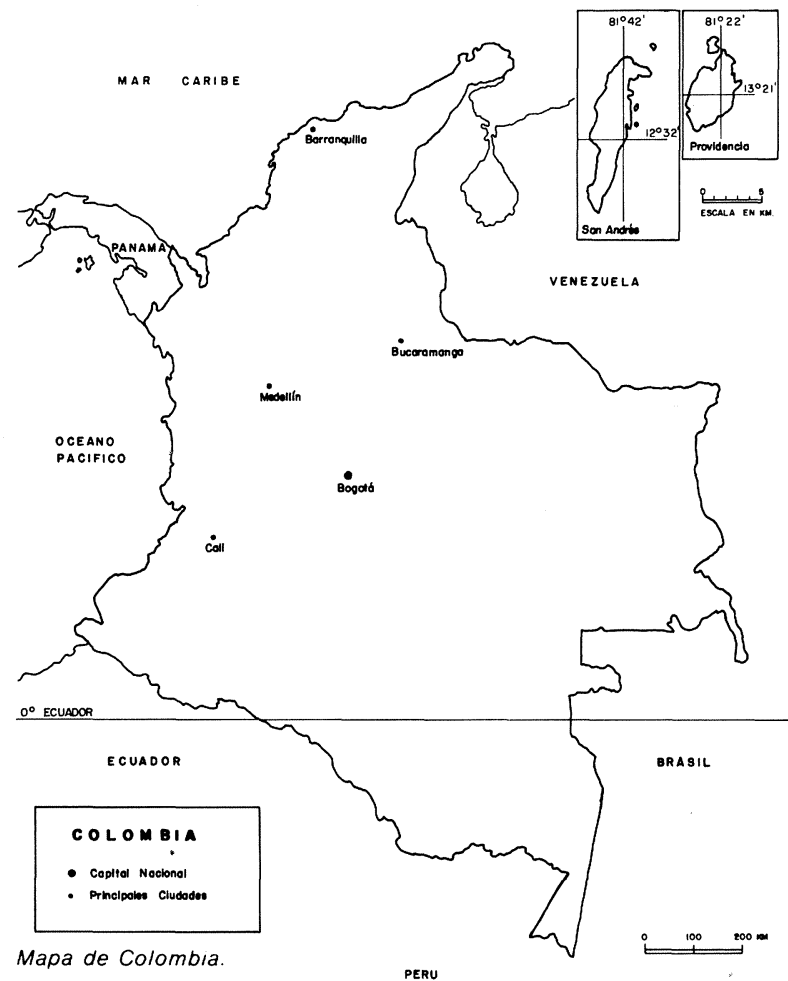

En Bogotá la población en los últimos 30 años han pasado de 658.000 habitantes a casi 5.000 .000 con un déficit en materia de vivienda que sobrepasa las 350.000 unidades.

Este hecho está particularmente marcado en la aparición y desarrollo continuo de asentamientos marginales y cobra mayor importancia cuando el fenómeno supera en el tiempo los métodos de planificación.

De otra parte como consecuencia directa de la falta de medios económicos, el número de viviendas producidas por las entidades gubernamentales 0 con ayuda de ellas es totalmente ineficaz y su trabajo se desarrolla en continuo desacuerdo con las autoridades planificadoras, quienes sencillamente ignoran el problema urbano de mayor magnitud.

Es así como en Bogotá, Cali, Medellín y Barranquilla (al igual que en las grandes ciudades del todo el mundo), más del $70 \%$ de las viviendas han sido construidas por el sector informal (particulares con ayuda del Gobierno), mediante sistemas de autoconstrucción y desarrollo progresivo; anotando en particular y como ejemplo, que en Bogotá durante los últimos años el $43 \%$ de las viviendas se han realizado en sectores marginales ilegales, fuera del perímetro urbano.

Lo anterior, sumado al encarecimiento y especulación del suelo urbano ha hecho extender los limites de la ciudad en forma desproporcionada, deformando las condiciones físicas normales de las poblaciones y elevando los costos de infraestructura a niveles difícilmente alcanzables.

Paralelo al fenómeno de dispersión de la ciudad, favorecido en la mayoría de los casos por las entidades oficiales, las áreas centrales se han venido deteriorando reemplazando sus usos y perdiendo el carácter de albergue habitacional. En plazos relativamente cortos se transforman de vivienda a áreas comerciales y posteriormente a bodegas, talleres, lotes vacios o inquilinatos $y$ asentamiento de viviendas marginales.

Esta doble condición, presente en todos nuestros centros urbanos, es motivo de continuos estudios, análisis y meta predilecta de los grandes proyectos de renovación y densificación, difíciles de concretar por la escasez de medios económicos y la zonificación social marcada por la especulación o simplemente desarrollada a partir de una errada concepción urbanistica.

En la última década se ha producido sobre todo en el sector económico y social, una revisión del concepto de vivienda urbana. Ante el circulo vicioso de aumento de usuarios atraídos por las perspectivas de grandes desarrollos oficiales y de la limitación, día a dia, de los medios económicos, se optó por regresar al usuario como principal agente de la construcción y desarrollo de la vivienda. Este hecho implica grandes transformaciones en el contexto urbano; en primer lugar los nuevos asentamientos aseguran una vida más continua, el desarrollo de las viviendas ocupa un mayor periodo de tiempo y un mejoramiento continuo con las siguientes ventajas de tipo económico. La intervención de los usuarios en las decisiones sobre planificación elimina en cierta forma los giros políticos y el cambio de planes e inversiones y proyectos. Finalmente la ciudad como medio complejo social regresa a sus origenes, para volverse a caracterizar de acuerdo a las condiciones culturales sociales y económicas de sus habitantes. 
Este hecho, enfrentado inicialmente en términos económicos toma fuerza en los medios urbanoarquitectónicos con los recientes planes de construcción de vivienda. Sin embargo es restringido a los sectores marginales de la ciudad que poco inciden en la imagen ideal de urbanistas y arqui- tectos conservando todos los vicios y traumas de los antiguos sistemas de planificación; es decir asumen la solución, pero no la enfrentan en forma integral, limitándola a la construcción de la "otra ciudad" y reduciendo los beneficios que ella conlleva.

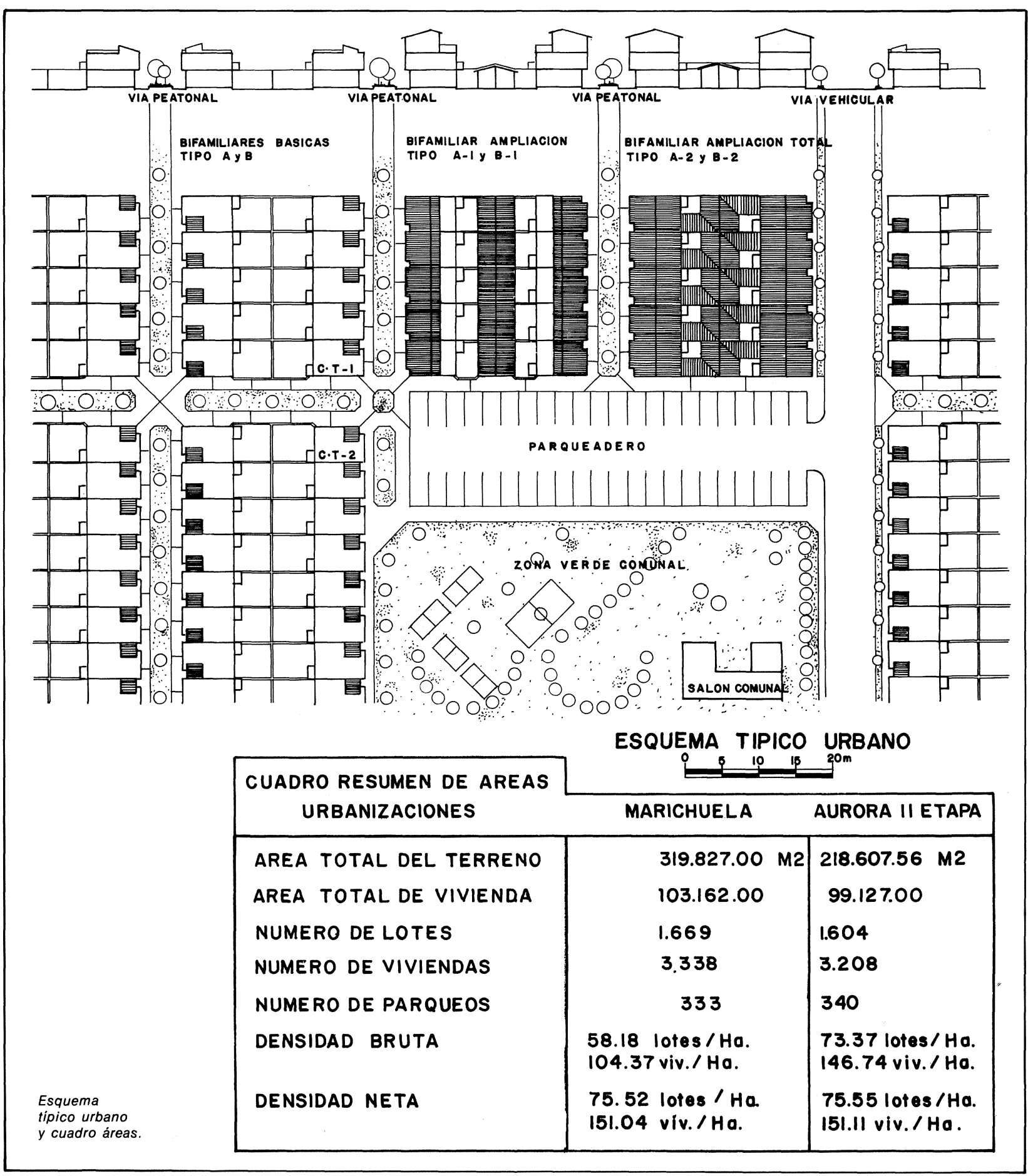


Mientras se habitan dudosos terrenos en la periferia de la ciudad a costos urbanos sumamente elevados las áreas centrales, poco habitadas, aumentadas en su valor, pero con las ventajas que su situación traduce, siguen en continuo deterioro impulsando la dispersión de las áreas urbanas.

La intervención popular en planes de ordenamien- to y en la construcción de viviendas, orientado con absoluta desconfianza hacia los desarrollos de los sectores marginales y periféricos pueden ser empleados en proyectos de renovación más viables y con más amplias perspectivas, sustrayendo necesariamente la imagen de proyectos terminados, propio de la urbanistica contemporánea.

\section{AURORA II - MARICHUELA/BOGOTA \\ DESARROLLO DE PROYECTO PROGRESIVO DE VIVIENDA DE INTERES SOCIAL, CON SISTEMAS TRADICIONALES E INDUSTRIALIZADOS}

Para poner en práctica esta política se constituyó un grupo profesional multidisciplinario que colabora estrechamente con el arquitecto proyectista en todas las etapas de su trabajo, lográndose asi que al término de éste, la unidad de vivienda sea arquitectónicamente buena, su construcción sea la mejor que se puede obtener por el dinero que se invierte y los elementos que intervienen en ella estén disponibles en el mercado en la cantidad y oportunidad necesarias para que la realización del programa se pueda llevar a cabo en un término suficientemente breve, para que los costos financieros no graven fuertemente el precio final de la vivienda.

Para controlar todo el desarrollo, tanto del proyec-
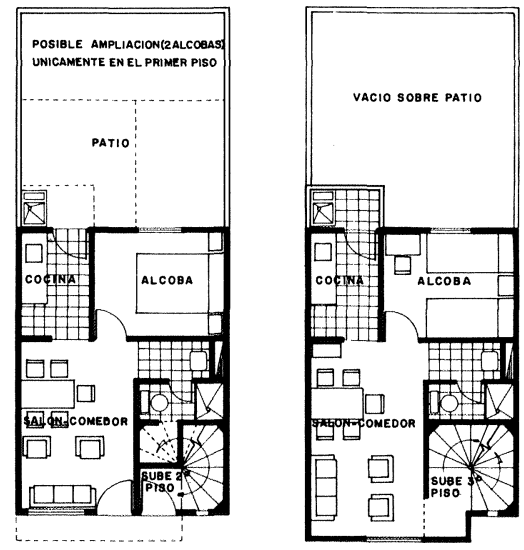

VIVIENDA TIPO B

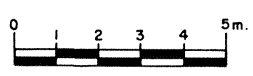

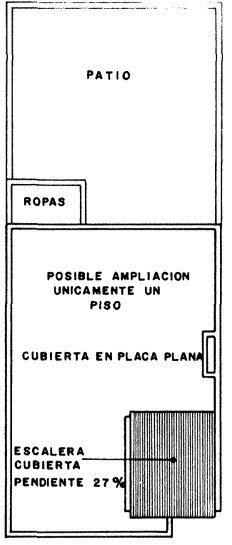

PLANTA de CUBiertas to arquitectónico y urbanístico, su gestión ante las autoridades, la consecución de financiaciones y la construcción de la urbanización y las viviendas, se emplean programas Gantt y CPM generados dentro del mismo grupo, usando o no computador, de acuerdo a las condiciones de cada uno de ellos.

Todo este proceso ha permitido llevar a cabo el proyecto de 6.500 unidades de vivienda en un año y su construcción a razón de 100 viviendas por semana en el siguiente año, con un empleo de 25 profesionales y 2.000 obreros directamente vinculados a la obra.
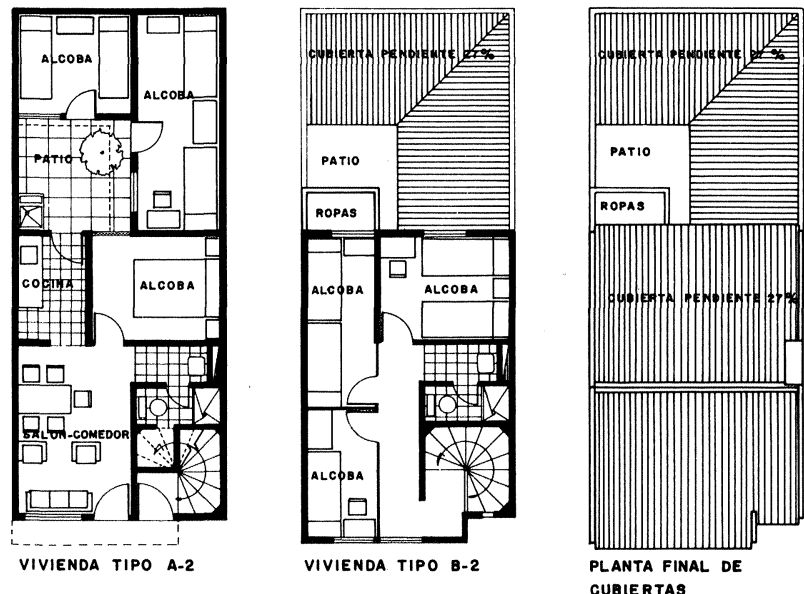

CUBIERTAS 


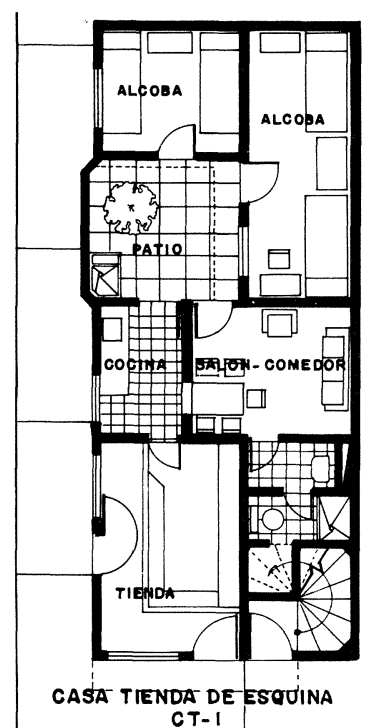

$C T-1$
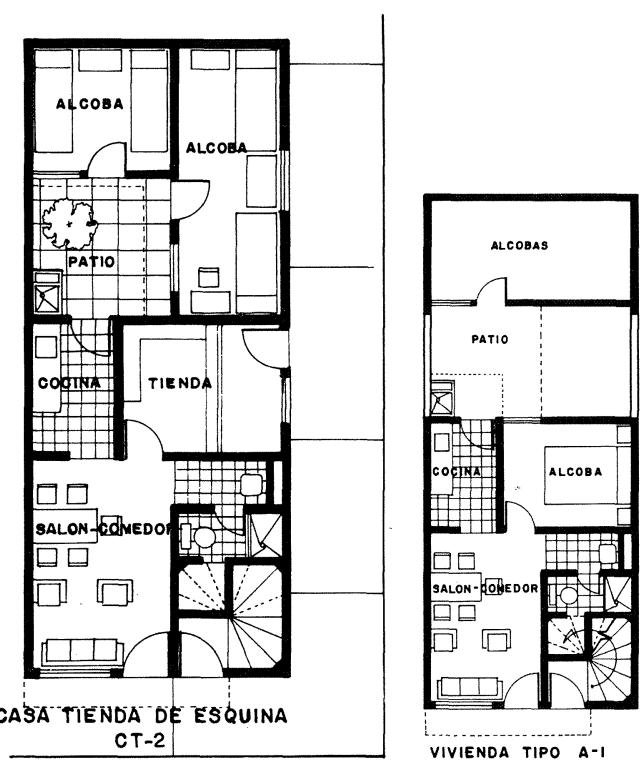

VIVIENDA TIPO A-I

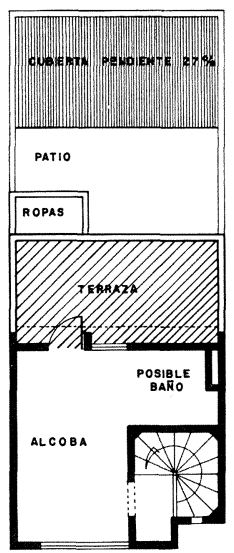

VIVIENDA TIPO $8-1$

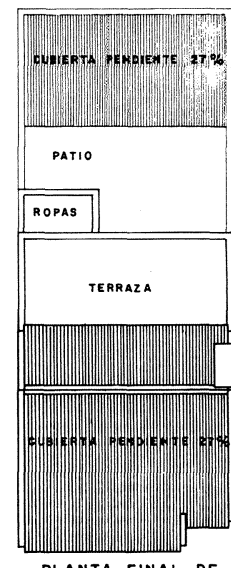

PLANTA FINAL DE

Planos vivienda tipo $A 1$ y $B$
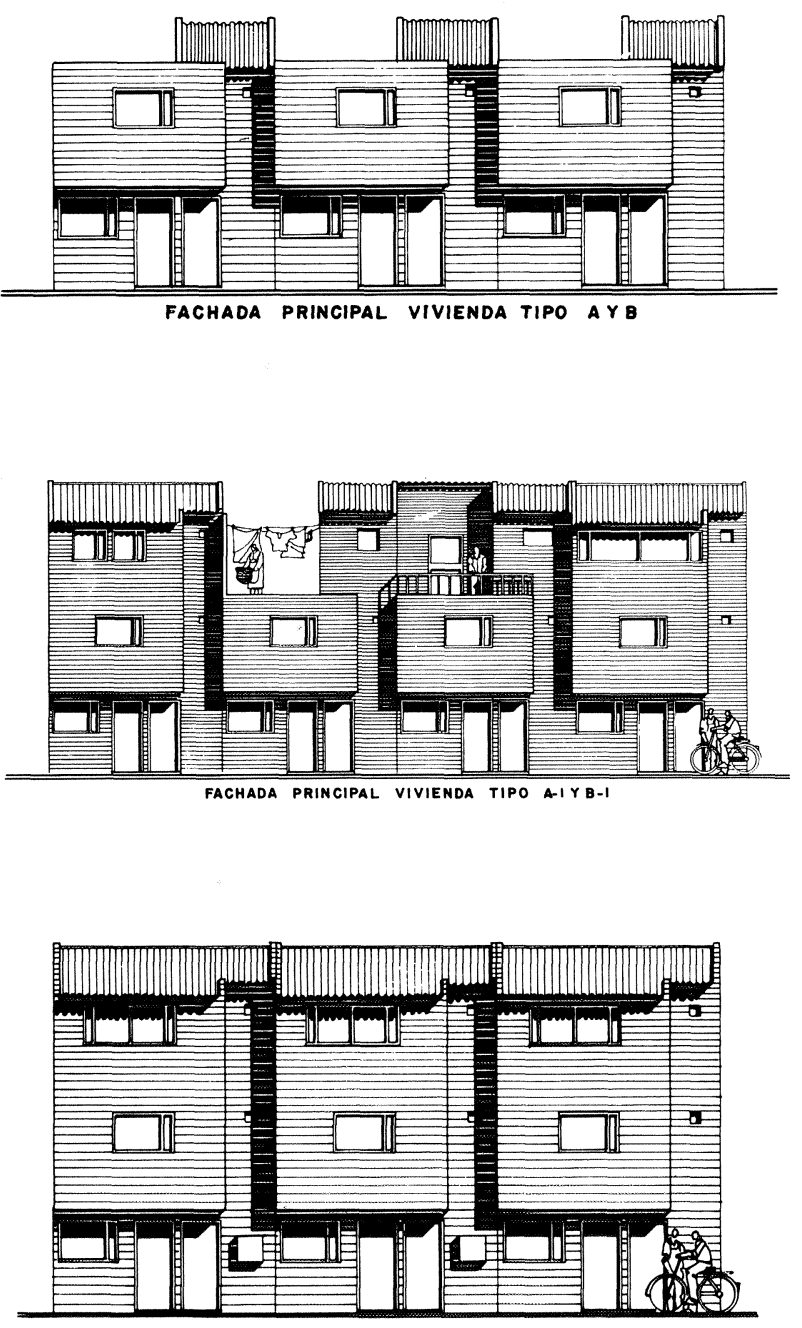

FACHADA PRINGIPAL VIVIENDA TIPO A-2 Y B-2

Elevaciones frontales
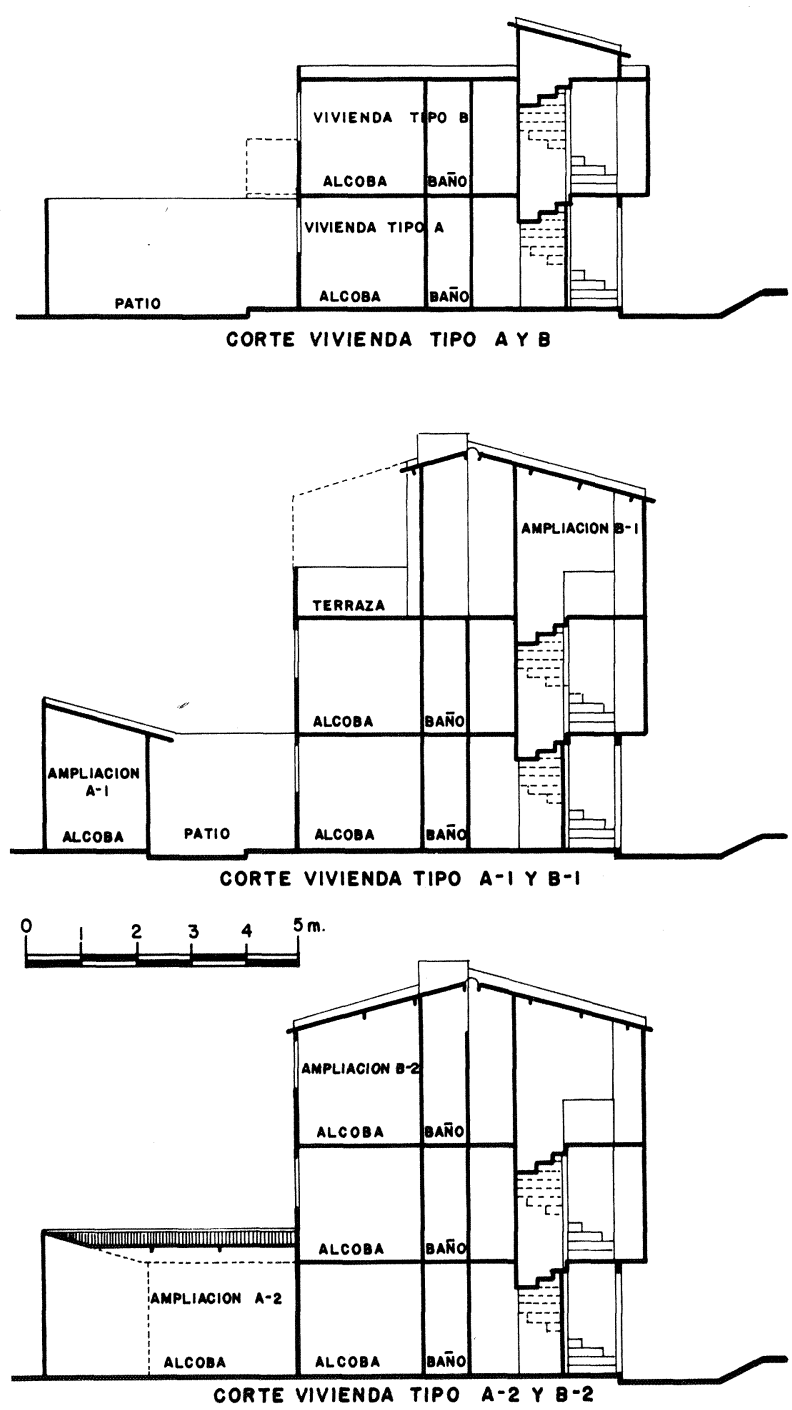

Cortes de viviendas $A, A 1, A 2$ y $B, B 1, B 2$. 


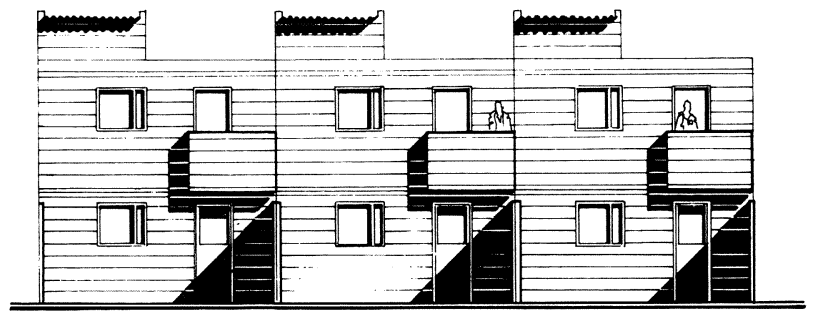

FACHADA POSTERIOR VIVIENDA TIPO A YB

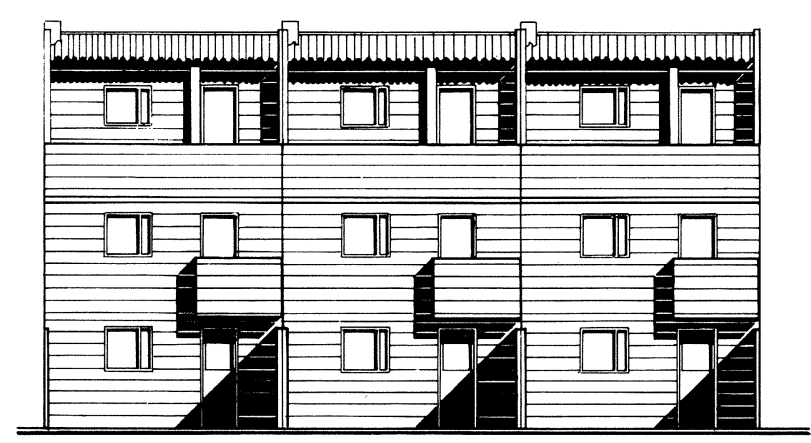

FACHADA POSTERIOR VIVIENDA TIPO A-I YB-I

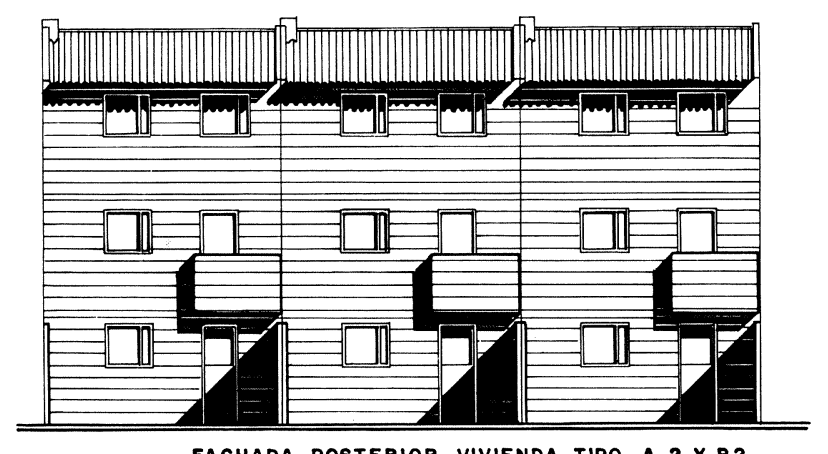

FACHADA POSTERIOR VIVIENDA TIPO A-2 Y B2

Elevaciones posteriores.

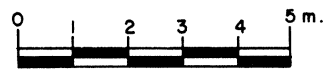

\section{BREVE DESCRIPCION DEL PROCESO DE CONSTRUCCION}

La base de organización es la misma de las cadenas de producción en serie. La construcción se ha dividido en actividades menores, de las cuales se encargan cuadrillas de obreros especializados $y$ que permanecen siempre en la misma actividad; estas actividades se reúnen en tres grupos, cada uno de los cuales está a cargo de un residente, haciéndose énfasis a este nivel como a todos los demás que la división de los encargos y responsabilidades está ligada al trabajo y no a accidentes geográficos. Una actividad sólo se considera terminada cuando se puede iniciar y realizar totalmente la siguiente. En todos los sistemas de contratación, control y pago se aplican estos principios básicos.
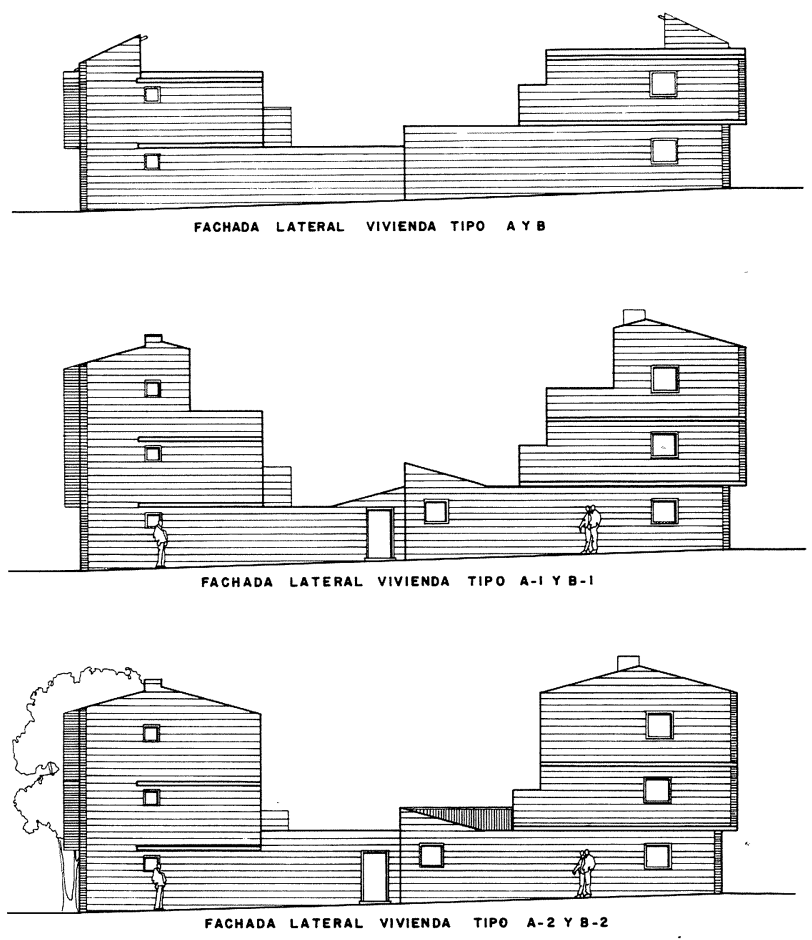

Elevaciones laterales.

\section{MATERIALES}

Ladrillo cerámico macizo en fachadas y aligerado en muros.

Placa de primer piso y cubierta maciza de $12 \mathrm{~cm}$ de espesor armada en dos sentidos con previsiones para ampliación.

Escalera prefabricada en hormigón (dos tramos).

Ventaneria en aluminio.

\section{LA VIVIENDA}

En un lote de terreno de $5 \mathrm{~m} \times 12,60 \mathrm{~m}^{2}$ (lote minimo según normas) se desarrolla el bifamiliar.

La vienda $A$ a nivel del terreno cuenta con area social, cocina, alcoba, y baño múltiple, puede ampliarse progresivamente en dos o tres alcobas $y$ un patio (ver planos).

La vivienda B con un programa similar al anterior se amplia en el tercer nivel hasta duplicar su área.

En ambos casos se entregan planos de posibilidades de ampliación y una cartilla de uso de la vivienda.

Existen soluciones especiales en las esquinas para tiendas y para lotes irregulares. 
El problema de la serie y monotonía de la repetición se soluciona dándole un volumen (voladizo) a cada unidad y con el aporte de la comunidad al producir ampliaciones con el mismo material, pero cambiando varios, haciendo balcones, etc., esto es apreciable hoy seis meses después de entregada la vivienda.

El sistema de bifamiliares fuera de aumentar la densidad produciendo vida en las calles; educa a los nuevos propietarios para la vida en comunidad antes de pasar a vivir en multifamiliares de varios pisos siguientes paso de muchos habitantes de estos barrios.

\section{LA SOLUCION URBANISTICA}

Dentro de las limitaciones económicas y de normas mínimas, estas dos urbanizaciones tienen como características básicas; a) Mínimo de perimetro de viales; b) Peatonales de 6 y 7,5 m de ancho y zonas comunes con algunos servicios comunales.

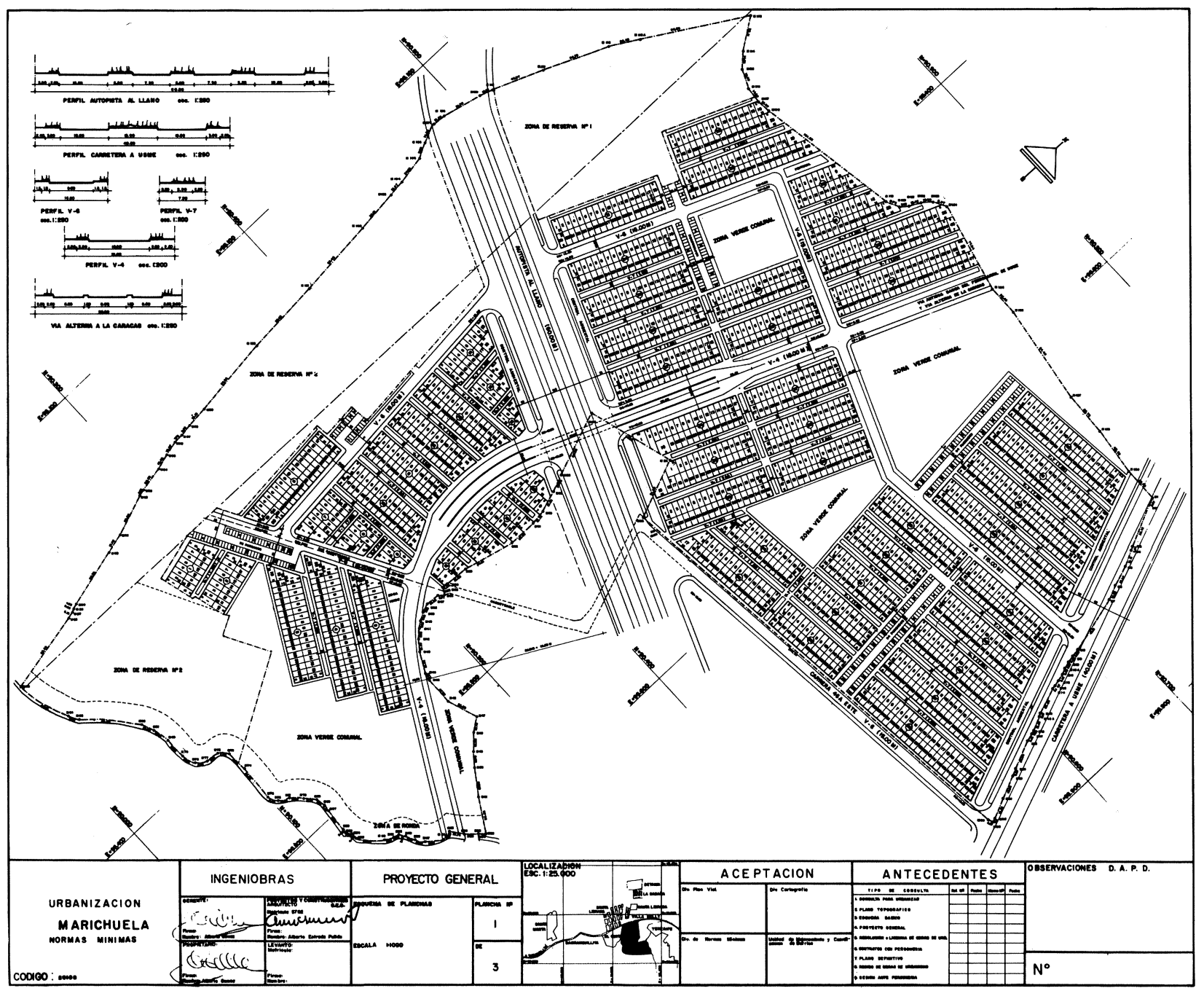

Plano urbanistico Marichuela. 


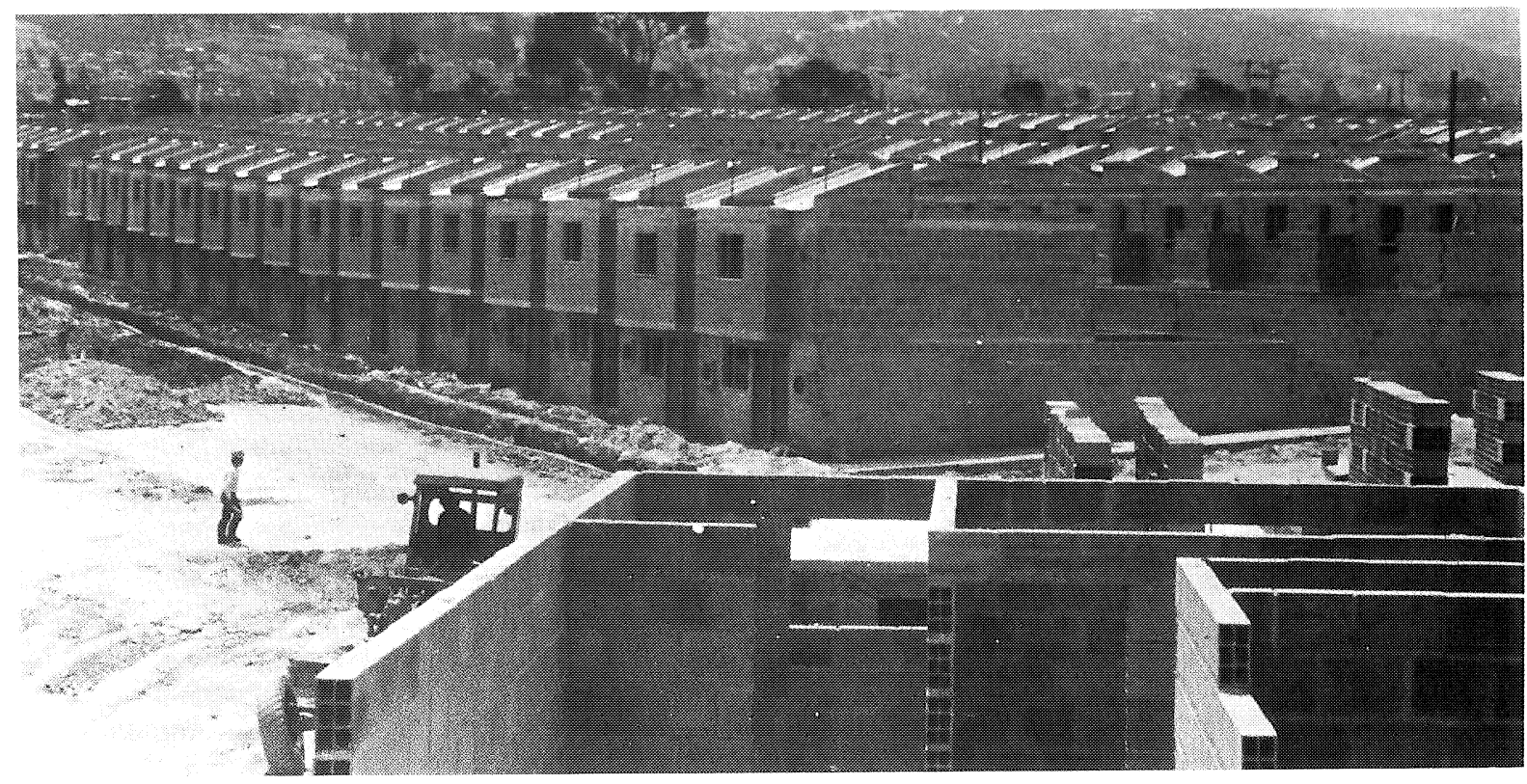

Foto general urbanizacion

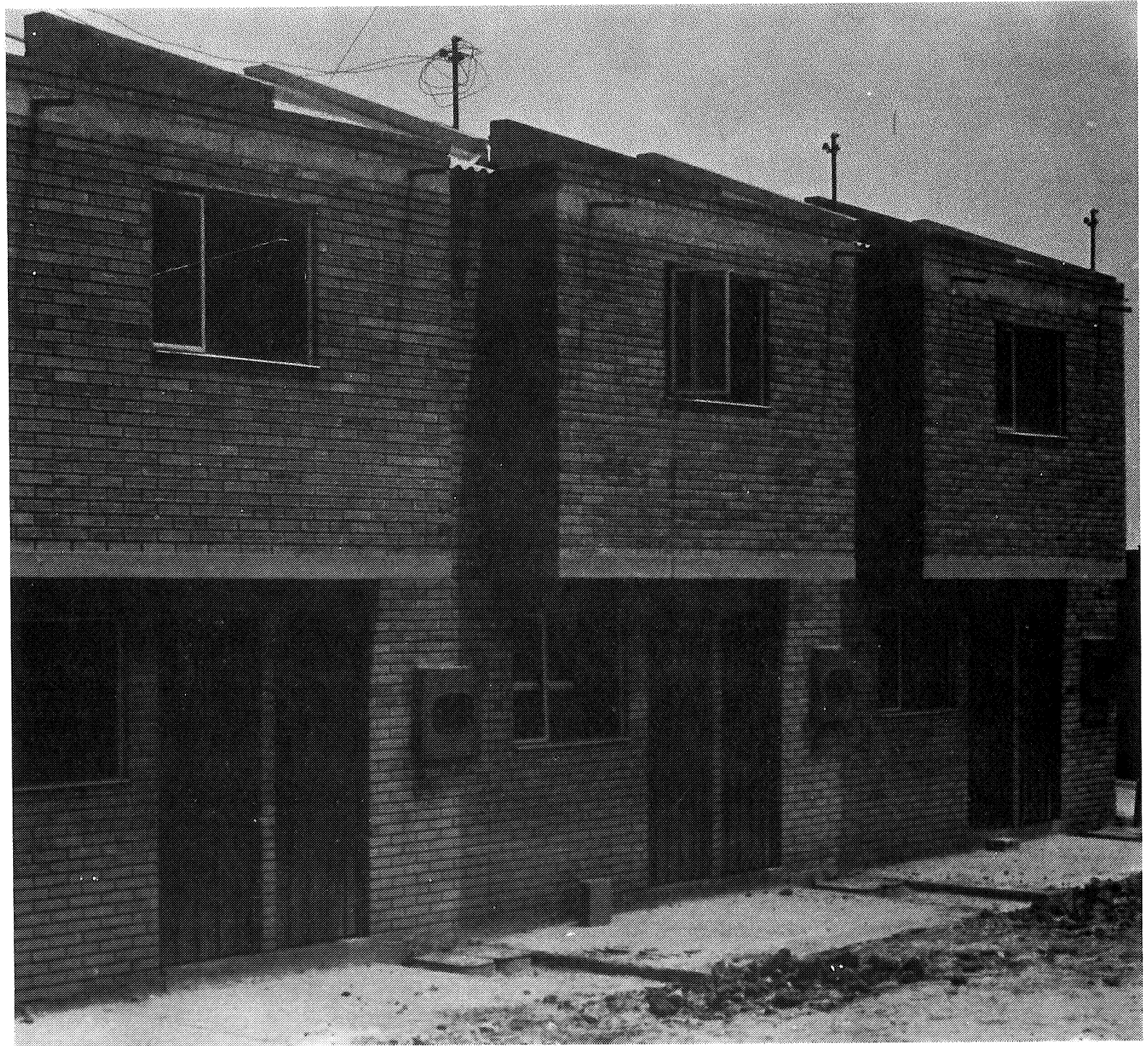

Foto particular vivienda 

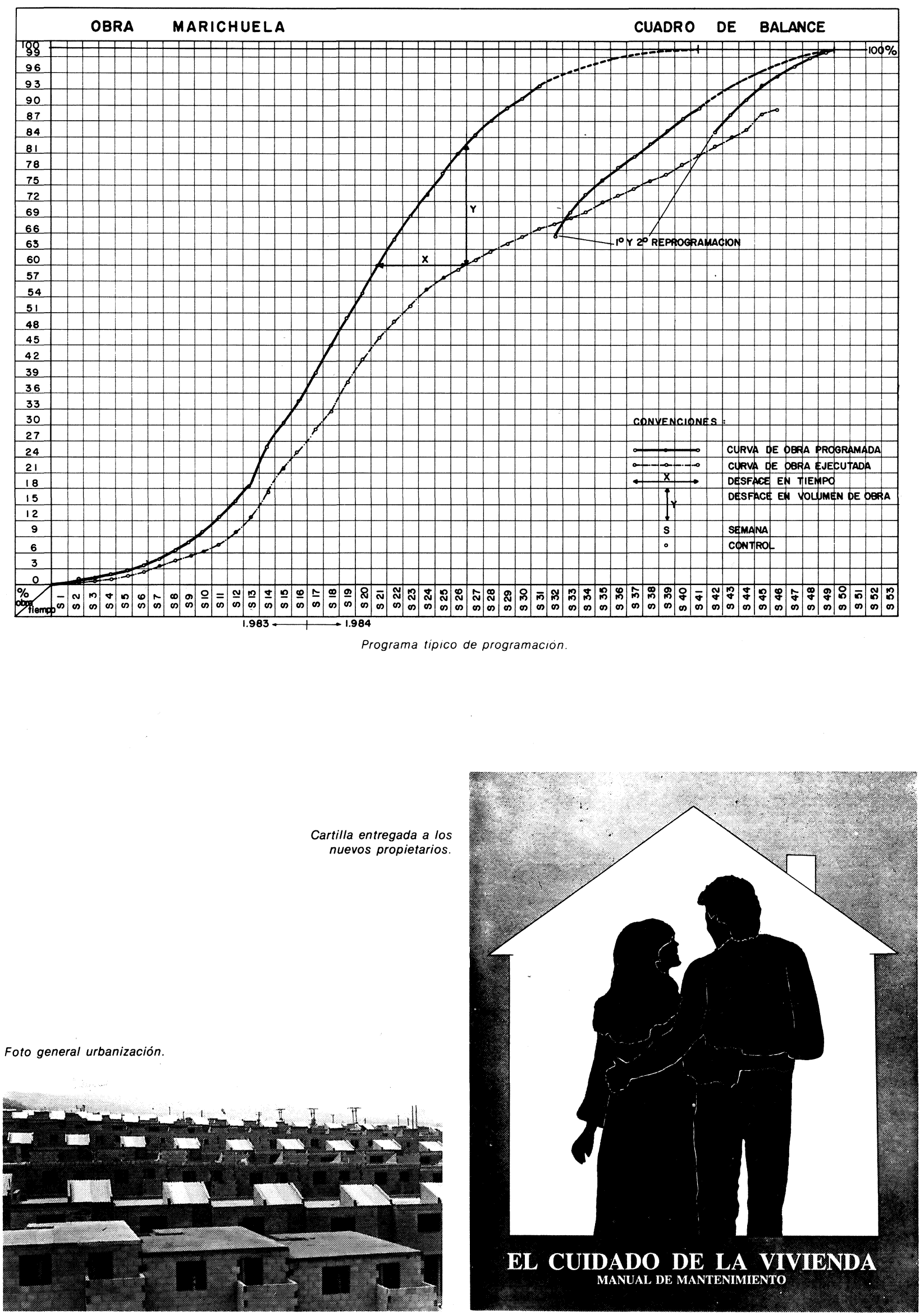